\title{
Symmetric Flat Connections, Triviality of Loi's Invariant and Orbifold Subfactors
}

By

\author{
Satoshi Goto*
}

\begin{abstract}
We define a notion of symmetric connections on subfactors and get a sufficient condition for a subfactor to have a symmetric connection. We also give a necessary and sufficient condition for Loi's invariant of a non-strongly outer automorphism of a subfactor to be trivial in the case with a symmetric connection. We apply this result to non-AFD $S U(n)$, subfactors and construct orbifold subfactors of non-AFD $S U(n)$, subfactors as well as the AFD case, as conjectured in our previous work. This generalizes constructions of Evans-Kawahigashi and $\mathrm{Xu}$.
\end{abstract}

\section{§. Introduction}

Orbifold construction coming from the technique in conformal field theory was first used in the subfactor theory by Y. Kawahigashi in [Ka1]. It is a method to construct new paragroups from paragroups with certain symmetry. He showed that subfactors of AFD factor of type $\mathrm{II}_{1}$ with principal graph $D_{2 m}$ are obtained from those with principal graph $A_{4 m-3}$ by taking simultaneous $\mathbf{Z}_{2}$-crossed products. The assumption of being AFD has later turned out to be not essential. Indeed, we generalized the orbifold construction to non-AFD subfactors with principal graph $A_{2 m+1}$ as well as in the AFD case by using bimodule technique in $[G]$. In these cases, non-strongly outer automorphisms on these subfactors with principal graph $A_{2 m+1}$ give symmetries of the connections. And results of the orbifold construction are determined by trivialities of Loi's invariants ([L]) of these non-strongly outer automorphisms and some values of certain partition functions which originally meant the flatness of a connection in [Kal].

D.E.Evans and Y.Kawahigashi generalized this orbifold construction to subfactors arising from $S U(n)$ solvable lattice models in the AFD case. (See [EK 1].) In this work, the flatness of the connections of the orbifold subfactors is also determined by the values of some partition functions. In general, it is very complicated and hard to compute these partition functions, but F. Xu overcame the difficulty by using the technique of conformal field theory and obtained the

Communicated by H. Araki, May 27, 1994.

1991 Mathematics Subject Classification(s): $94-42$

* Department of Mathematical Sciences, University of Tokyo. Komaba. Tokyo 153, Japan. 
values of them in $[\mathrm{X} 1]$. He showed that the values of these partition functions are the exponentials of the conformal dimensions.

We conjectured in $[G]$ that we can also apply the orbifold method to nonAFD $S U(n)_{k}$ subfactors by using the values of these partition functions. We will prove this conjecture in this paper with a new general method. To solve this problem, first, we have to take the connections of the subfactor symmetric, because the values are not well-defined unless we do so. So we define a notion of symmetric connections and give a sufficient condition for a subfactor to have a symmetric connection. The next important problem is triviality of Loi's invariant of the non-strongly outer automorphism on the subfactor. In the case of subfactors with principal $A_{2 m+1}$, it was trivial because the higher relative commutants are generated by Jones' projections. But in the case of $S U(n)_{k}$ subfactors, triviality of Loi's invariant is not trivial. So we deal with this problem in more general situation and give a necessary and sufficient condition for it. This result is also related to the relative Connes invariant

$$
\chi(M, N)=\frac{\mathrm{Ct}(M, N) \cap \overline{\operatorname{Int}}(M, N)}{\operatorname{Int}(M, N)}
$$

for subfactors introduced in [Ka2]. Popa [P1], [P2] has shown that the central triviality of automorphisms of strongly amenable subfactors of type $\mathrm{II}_{1}$ is equivalent to their non-strong outerness. It has been known by Loi [L] that the approximate innerness of an automorphism of a strongly amenable subfactor of type $\mathrm{II}_{1}$ is characterized by triviality of his invariant [L]. Thus it is necessary to determine which non-strongly outer automorphisms of a subfactor have the trivial Loi invariant, in order to compute $\chi(M, N)$ for strongly amenable subfactors. Our result here gives a solution to this problem.

In section 3, we apply our result to non-AFD $S U(n)_{k}$ subfactors in Theorem 3.2 and Theorem 3.4. This result is a generalization of Theorem 3.9 in [G], i.e., in the case of $n=2$, we can get non-AFD subfactors with principal graphs $D_{2 m}$ from those with principal graphs $A_{4 m-3}$ by taking simultaneous $\mathbb{Z}_{2}$-crossed products. We will also apply our method to generalize $\mathrm{Xu}$ 's computation [X2] of the flat parts of non-flat orbifold constructions to non-AFD case. Even in the AFD case, our proof is simpler than Xu's.

Note that our result can be applied to non-AFD $S U(n)_{h}$ subfactors of type $\mathrm{II}_{1}$ obtained from the construction of S. Popa [P3] and F. Rădulescu [R].

In this paper, we assume that all the factors are of type $\mathrm{II}_{1}$ for simplicity. But the exactly same method works also for $S U(n)_{k}$ subfactors of type $\mathbb{I I}_{\infty}$ and type III, if we use the general bimodule theory. (See [Y1], [Y2].)

The author is grateful to Professor Y. Kawahigashi for constant encouragement and many important suggestions. 


\section{$\S 1$ Non-strongly Outer Actions on Subfactors}

In this section we discuss non-strongly outer actions on subfactors. We refer readers to [O1], [O2] and [O3] for a notion of connections and the notations. We also refer readers to $[\mathrm{CK}]$ and $[\mathrm{Ko}]$ for a notion of strong outerness of automorphisms. We use the same notations as in [G]. In the present paper, we mainly deal with the principal graphs of subfactors in order to get the triviality of Loi's invariant. This is a different point of the previous paper $[G]$, where we used the dual principal graph. So we need the following theorem and can easily prove it in the same way as in the proof of Theorem 2.2 in [G].

Theorem 1.1. (Choda-Kosaki, [CK, Theorem 2], [Ko, Theorem 3])

Let $\alpha \in \operatorname{Aut}(M, N)$ and $\beta=\left.\alpha\right|_{N}$. Then the following are equivalent.

(i) There exists a non-zero element $a \in M_{h}$ such that an $=\alpha(n)$ a for all $n \in N$.

(ii) ${ }_{N}\left(M_{k}\right)_{N} \succ{ }_{N}\left({ }_{\beta} N\right)_{N}$.

Remark. In the above theorem we can regard an element $a \in M_{h}$ with $a n=$ $\alpha(n) a$ for all $n \in N$ as an element $a \in \operatorname{Hom}\left({ }_{N}\left(M_{l}\right)_{M},_{N}\left({ }_{\alpha} M_{t}\right)_{M}\right)$ if $k=2 i$ is even, $\left(a \in \operatorname{Hom}\left({ }_{N}\left(M_{1}\right)_{N},_{N}\left({ }_{\alpha} M_{t}\right)_{N}\right)\right.$ if $k=2 i+1$ is odd). Then we have the relation that $a$ is equal to $\tilde{a}(\hat{1})$ up to scalar multiplication. Here $\tilde{a}$ means the Frobenius dual of

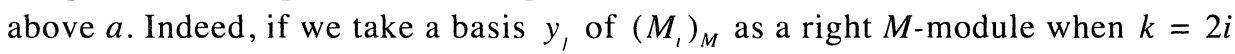
is even,

$$
\begin{aligned}
\tilde{a}(\hat{1}) & =[N: N]^{1 / 2}\left[M_{1}: M\right]^{-1 / 2} \sum_{1} a\left(\hat{l} \otimes_{N} y_{1}\right) \otimes_{M} y_{1} \\
& =\left[M_{1}: M\right]^{-1 / 2} \sum_{1} a y_{1} \otimes_{M} y_{1} \\
& =\left[M_{1}: M\right]^{-1 / 2} a
\end{aligned}
$$

And similarly we can show the same relation when $k=2 i+1$ is odd. This relation plays an important role later.

Corollary 1.2. For all $x \in N^{\prime} \cap M_{h}$ there exists an element $\xi \in$ $\operatorname{Hom}\left({ }_{N}\left(M_{h}\right)_{N}, N_{N}\right)$ such that $\xi^{\prime}(\hat{1})=x$.

Let $N \subset M$ be a pair of factors of type $\mathrm{II}_{1}$ with finite index and with trivial relative commutant. We denote its principal graph by " and denote its dual principal graph by $\dddot{~}$. So we have the following graph

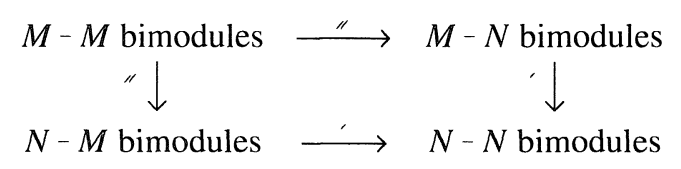


We also denote the vertices of the graph $\xi$ by $\mathscr{\xi}^{(0)}$ and the edges of the graph " by $\mathscr{G}^{(1)}$. For the graph $\mathscr{H}$, we use similar notations.

We identify a vertex $X \in \mathscr{G}^{(0)}$ with the corresponding bimodule. An (oriented) horizontal (resp. vertical) edge $\xi \in \mathscr{G}^{(1)}$ with source vertex (bimodule) $X \in \mathscr{G}^{(0)}$ and range vertex (bimodule) $Y \in \mathscr{G}^{(0)}$ represents an intertwiner $\xi \in$ $\operatorname{Hom}(X \otimes M, Y)$ (resp. $\xi \in \operatorname{Hom}(M \otimes X, Y))$. Here $M$ represents the bimodule ${ }_{M} M_{N}$ or ${ }_{N} M_{M}$.

First of all, we assume the following conditions (A1) and (A2).

(A1) $\mathscr{G}$ and $\mathscr{H}$ are finite, i.e., the subfactor $N \subset M$ has finite depth.

(A2) there exist non-trivial automorphisms $\alpha \in$ Aut $M$ and $\beta \in$ Aut $N$ such

that ${ }_{M}\left({ }_{\alpha} M\right)_{M} \in \mathscr{H}^{(0)},{ }_{N}\left({ }_{\beta} N\right)_{N} \in \mathscr{G}^{(0)}, \quad$ and ${ }_{M}\left({ }_{\alpha} M\right)_{M} \otimes_{M} M_{N} \cong{ }_{M} M_{N} \otimes_{N}\left({ }_{\beta} N\right)_{N}$ i.e., $\alpha \mathrm{I}_{N}=\beta$.

In this case $\alpha$ and $\beta$ are non-strongly outer automorphisms and appear in the same depth of the graphs $\mathscr{H}^{\prime}$ and $\mathscr{G}$. From condition (A1), there exists an integer $n \in \mathbb{N}$ such that ${ }_{M}\left({ }_{\alpha^{n}} M\right)_{M} \cong_{M} M_{M}$, that is, $\alpha^{n} \in \operatorname{Int} M$. We denote the outer period of the automorphism $\alpha$ by $n$. Now we also assume the following condition.

(A3) $\beta^{n}=1_{N}$ with the same integer $n$ as above.

We claim in the next lemma that condition (A3) follows from the following conditions (A4) and (A5).

(A4) $\beta^{n} \in \operatorname{Int} N$ with the same integer $n$ as above, i.e., $\alpha$ and $\beta$ have the same outer periods.

(A5) there exists an $N-N$ or $N-M$ bimodule $Y \in "(0)$ such that $Y \cong_{\beta} Y$.

Lemma 1.3. (cf. [I, Lemma 3.3]) If we have conditions (A4) and (A5), then we can choose a representative of $\beta$ so that $\beta^{n}=1_{N}$.

Proof. Suppose there exists an $N-N$ bimodule $Y$ such that ${ }_{N} Y_{N} \cong_{N}\left({ }_{\beta} Y\right)_{N}$. Set $P \equiv \operatorname{End}\left(Y_{N}\right)$. Then there exists a unitary $u \in \| /(P)$ such that $u \in \operatorname{Hom}\left({ }_{N} Y_{N}\right.$, $\left.{ }_{N}\left({ }_{\beta} Y\right)_{N}\right)$. So the unitary $u$ satisfies the following.

$$
u(n \cdot y)=n \cdot u(y)=\beta(n) u(y), \text { for all } n \in N \text { and } y \in Y .
$$

So $\beta(n)=u n u$ for all $n \in N$. Now from the condition (A4), there exists a unitary $v \in \mathscr{H}(N)$ such that $\beta^{n}=\operatorname{Ad} u^{n}=\operatorname{Ad} v$. We have $N^{\prime} \cap P=\mathbb{C}$ because of the irreducibility of ${ }_{N} Y_{N}$, so there exists a scalar $c \in \mathbb{C}$ such that $u^{n}=c v$ and

$$
u^{n}=u u^{n} u=u(c v) u=c \beta(v),
$$

hence $\beta(v)=v$. So the obstruction of $\beta$ is trivial (cf. [C]). Therefore, there exists a unitary $w \in \mathbb{R}(N)$ such that $(\operatorname{Ad} w \circ \beta)^{n}=1_{N}$.

In the case when there exists an $N-M$ bimodule ${ }_{N} Y_{M}$ such that ${ }_{N} Y_{M} \cong_{N}\left({ }_{\beta} Y\right)_{M}$, we can prove the lemma in the same way.

Q.E.D. 
Corollary 1.4. If we have condition (A3) (or (A4) and (A5)), we can take $\alpha$ and $\beta$ so that

$$
\alpha^{n}=1_{M}, \quad \beta^{n}=1_{N}, \quad \alpha I_{N}=\beta
$$

Proof. From conditions (A2), (A3) and Lemma 1.3 we can take $\alpha$ and $\beta$ so that

$$
\alpha^{n} \in \operatorname{Int} M, \quad \alpha \mathrm{I}_{N}=\beta, \quad \beta^{n}=1_{N},
$$

and in this case $\alpha^{n}=1_{M}$ because $N^{\prime} \cap M=\mathbf{C}$.

Q.E.D.

From the above corollary, the automorphism $\alpha$ becomes a $\mathbf{Z}_{n}$-action on the subfactor $N \subset M$. So we can consider the simultaneous crossed product subfactor

$$
N \rtimes_{\alpha} \mathbf{Z}_{n} \subset M \rtimes_{\alpha} \mathbf{Z}_{n} .
$$

We call this new subfactor the orbifold subfactor of $N \subset M$. It is well known that Jones' tower of this new subfactor is the following,

$$
N \rtimes_{\alpha} \mathbf{Z}_{n} \subset M \rtimes_{\alpha} \mathbf{Z}_{n} \subset M_{1} \rtimes_{\alpha} \mathbf{Z}_{n} \subset \cdots \subset M_{h} \rtimes_{\alpha} \mathbf{Z}_{n} \subset \cdots .
$$

By computing the higher relative commutants $\left(N \rtimes_{\alpha} \mathbf{Z}_{n}\right)^{\prime} \cap\left(M_{k} \rtimes_{\alpha} \mathbf{Z}_{n}\right)$, we obtain the (dual) principal graph of this orbifold subfactor.

\section{§2. Symmetric Connections and Triviality of Loi's Invariant}

To compute the higher relative commutants of the orbifold subfactors, we need a symmetry of the connection.

Let $N \subset M$ be a pair of factors of type $\mathrm{II}_{1}$ with principal graph $\because$ and dual principal graph $\mathbb{H}$. As in the section 3 , we use the same symbols $\because(0), Z^{(1)}$ and so on.

Definition 2.1. A subfactor $N \subset M$ of type $\mathrm{II}_{1}$ is said to have a symmetric connection if there exist a graph automorphism $\sigma$ and a choice of

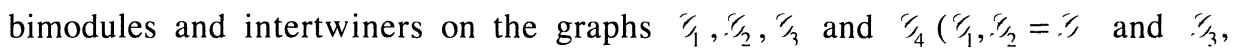
$\overline{3}_{4}=\mathbb{1}$ ) satisfying the following condition.

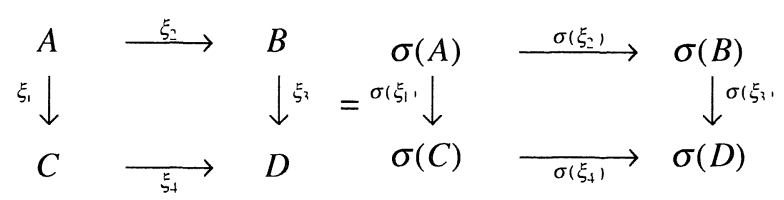

In this case, a connection of the subfactor $N \subset M$ with the graph automorphism $\sigma$ satisfying the above condition is called a symmetric connection. Moreover if there exists a natural number $n$ such that $\sigma^{n}=i d$ " 


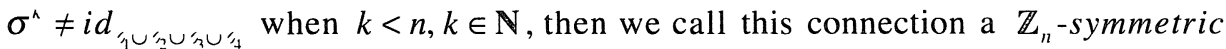
connection. It is clear that if a subfactor $N \subset M$ has a $\mathbb{Z}_{n}$-symmetric connection and $n=m k$ for some $m, k \in \mathbb{N}$, then it has a $\mathbb{Z}_{m}$-symmetric connection because we may take $\tau \equiv \sigma^{k}$ as the symmetric graph automorphism.

Let $N \subset M$ be a pair of factors of type $\mathrm{II}_{1}$ which satisfies assumptions (A1) $\sim$ (A3). Then we can regard the non-strongly outer automorphism $\alpha$ (resp. $\beta$ ) as a $\mathbb{Z}_{n}$-symmetric graph automorphism on the graph $\mathscr{H}^{\prime}=\mathscr{F}_{4}$ (resp. $\mathscr{G}=\mathscr{E}_{2}$ ) as follows. We define the mapping $g_{\alpha}$ on the graph $\mathscr{H}$ as follows,

$$
\begin{aligned}
& \text { as a mapping on } \mathscr{H}^{(0)} \text {, we define } \begin{cases}g_{\alpha}(X) \equiv_{\alpha} X & \text { if } X{\equiv_{\alpha}}_{\alpha} X \\
g_{\alpha}(X) \equiv X & \text { if } X \cong_{\alpha} X\end{cases} \\
& \text { and as a mapping on } \mathscr{H}^{(1)}, g_{\alpha}(\xi) \in \mathscr{H}_{g_{\alpha}(X), g_{\alpha}(Y)}^{(1)} \text { if } \xi \in \mathscr{H}_{X, Y}^{(1)}\left(X, Y \in \mathscr{H}^{(0)}\right) \text {, }
\end{aligned}
$$

then $g_{\alpha}$ is a $\mathbb{Z}_{n}$-symmetric graph automorphism because $\alpha^{n}=1_{M}$ and it has inverse mapping $g_{\alpha^{-1}}$. Here the symbol $\mathscr{H}_{X, Y}^{(1)}$ means the set of edges (intertwiners) of the graph $\mathscr{H}^{\prime}$ with source vertex (bimodule) $X$ and range vertex (bimodule) $Y$. Note that the number of edges in $\mathscr{H}_{X . Y}^{(1)}$ is equal to the dimension of the intertwiner space $\operatorname{Hom}(X \otimes M, Y)$. And we take these intertwiners so that they make an orthogonal basis. So if the graph $\mathscr{H}^{\prime}$ has some multilines, then we defines the above graph automorphism $g_{\alpha}$ so that it is a one-to-one map between these multilines. It is obvious that we can define $g_{\alpha}$ in such a way because $\operatorname{dim} \operatorname{Hom}(X \otimes M, Y)$ is equal to $\operatorname{dim} \operatorname{Hom}\left({ }_{\alpha} X \otimes M,{ }_{\alpha} Y\right)$.

In the case of the graph $\%=\frac{1}{2}$, we can define a $\mathbb{Z}_{n}$-symmetric graph automorphism $g_{\beta}$ in the same way. Note that these mappings $g_{\alpha}$ and $g_{\beta}$ are defined only on the horizontal edges. So in order to deal with a symmetric connection we also have to define the mapping on the vertical edges. We also note that the horizontal paths and the vertical paths are not symmetric in our graphical representation. Because we pass to horizontal direction by tensoring $M$ (more precisely ${ }_{M} M_{N}$ or ${ }_{N} M_{M}$ ) from the right, but to vertical direction by tensoring $M$ from the left as in the following diagrams,

$$
X \stackrel{\otimes M}{\longrightarrow} Y, \quad\left(\begin{array}{rr}
X \\
\text { resp. } & M \otimes \\
& Z
\end{array}\right),
$$

where $M$ is either ${ }_{M} M_{N}$ or ${ }_{N} M_{M}$. So we label the edges for these graph in the following way.

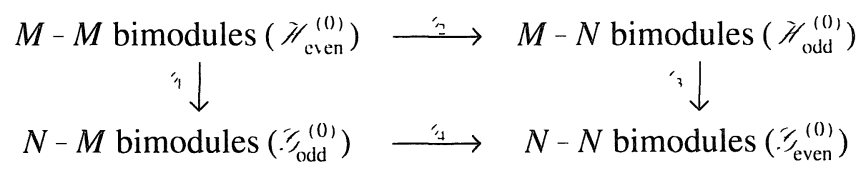

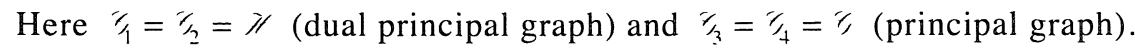


Consider the following condition (A6).

(A6) For each bimodule $X \in \varepsilon^{(0)}$ and $Y \in \mathscr{H}^{(0)}$, the sets of bimodules $\left\{_{\beta^{h}} X\right\}_{h=0}^{n-1}$ and $\left\{_{\alpha^{h}} Y\right\}_{h=0}^{n-1}$ have 1 or $n$ different equivalence classes. And any

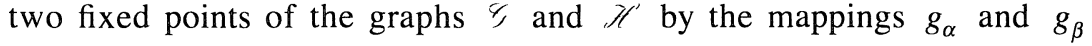
are not connected.

This condition is not essential, but we assume this for technical simplicity. Now we have the following proposition.

Proposition 2.2. If a subfactor $N \subset M$ of type $\mathrm{II}_{1}$ with finite index satisfies condition (A1) (A3) and (A6), then it has a $\mathbb{Z}_{n}$-symmetric connection.

Proof. (Step I) A construction of the $\mathbb{Z}_{n}$-symmetric graph automorphism $\sigma_{\alpha}$. We define the graph automorphism $\sigma_{\alpha}$ on the both graph $\mathscr{H}$ and $\mathscr{H}$ as follows. First we define $\sigma_{\alpha}=g_{\beta}$ on $"(0)$ and $\sigma_{\alpha}=g_{\alpha}$ on $\dddot{*}^{(0)}$.

Next we define it on the horizontal edges, i.e., on the graph $/ 2$ and $1 / 4$ so that it has the following property. We take $\xi \in \mathscr{H}^{(1)}$ and $s(\xi)=X, r(\xi)=Y,(X, Y \in$ ${ }^{\prime}\left({ }^{(0)}\right)$. Here $s(\xi)$ represents the source bimodule of $\xi$ and $r(\xi)$ represents the range bimodule of $\xi$. We take the following intertwiners.

$$
\begin{cases}\xi: X \otimes M \rightarrow Y, & \xi^{\prime} \equiv \xi:_{\alpha} X \otimes M \rightarrow_{\alpha} Y, \\ u: X \rightarrow_{\alpha} X, & v: Y \rightarrow_{\alpha} Y .\end{cases}
$$

Here $u$ and $v$ are surjective isometries between the two bimodules. We may and do take $u$ and $v$ so that $u^{n}=1$ and $v^{n}=1$. Then $\sigma_{\alpha}$ is defined by the following.

$$
\sigma_{\alpha}(\xi)= \begin{cases}\xi^{\prime}, & \text { if neither } X \text { nor } Y \text { is not fixed by } \sigma_{\alpha} \\ \xi^{\prime} \circ u, & \text { if } X \text { is fixed by } \sigma_{\alpha} \\ v \circ \xi^{\prime}, & \text { if } Y \text { is fixed by } \sigma_{\alpha} .\end{cases}
$$

In the case of the graph $/ \frac{1}{4}$, we define the mapping $\sigma_{\alpha}$ so that it satisfies the same property as above.

Finally we define $\sigma_{\alpha}$ on the vertical edges, i.e., on the graph $/ 1$ and $/ / 2$ as follows. We take $\eta \in \zeta^{(1)}$ or $\mathscr{H}^{(1)}$ and $s(\eta)=X, r(\eta)=Y,\left(X, Y \in \mathscr{G}^{(0)}\right.$ or $\left.\mathscr{H}^{(0)}\right)$. We take the following intertwiners.

$$
\begin{cases}\eta: M \otimes X \rightarrow Y, & \eta\left(\alpha \otimes 1_{X}\right): M \otimes_{\alpha} X \rightarrow_{\alpha} Y, \\ u: X \rightarrow_{\alpha} X, & v: Y \rightarrow_{\alpha} Y .\end{cases}
$$

Here ${ }_{\alpha} X$ (resp. ${ }_{\alpha} Y$ ) represents ${ }_{\beta} X$ (resp. ${ }_{\beta} Y$ ) if the bimodule $X$ (resp. $Y$ ) is a left $N$-module. Then $\sigma_{\alpha}$ is defined by the following. 


$$
\sigma_{\alpha}(\eta)= \begin{cases}\eta\left(\alpha \otimes 1_{X}\right), & \text { if neither } X \text { nor } Y \text { is not fixed by } \sigma_{\alpha} \\ \eta(\alpha \otimes u), & \text { if } X \text { is fixed by } \sigma_{\alpha} \\ \nu \circ \eta\left(\alpha \otimes 1_{X}\right), & \text { if } Y \text { is fixed by } \sigma_{\alpha} .\end{cases}
$$

Because the both graph $\mathscr{G}$ and $\mathscr{H}$ are finite we can construct this mapping in finite procedure and we can easily verify that this definition is well-defined. In order to verify the well-definedness of this mapping we have only to check the following conditions.

$$
\begin{gathered}
{[\eta(\alpha \otimes 1)]^{-}=\tilde{\eta}(\alpha \otimes 1)} \\
{[\eta(\alpha \otimes u)]^{-}=u \quad \circ \tilde{\eta}(\alpha \otimes 1)} \\
{[v \circ \eta(\alpha \otimes 1)]^{-}=\tilde{\eta}(\alpha \otimes v) .}
\end{gathered}
$$

Where $\tilde{\eta}$ is the Frobenius dual of $\eta$. We can easily check these by writing the Frobenius dual explicitly as in [O3] and [Y1].

(Step II) Symmetry of the connection.

Now we prove that the choice of bimodules and intertwiners such that the graph automorphism $\sigma_{\alpha}$ satisfies the above property makes the connection of this subfactor symmetric. From the above construction of $\sigma_{\alpha}$, the period of the symmetry is obviously $n$. We will show the following equality.

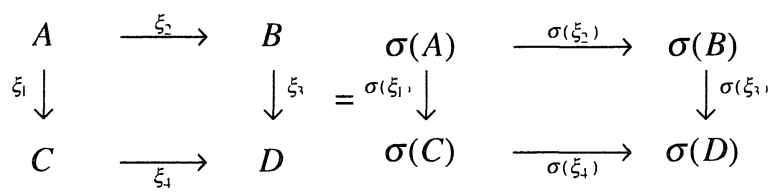

(i) In the case where any of the four bimodules $A, B, C, D$ is not fixed by $\sigma_{\alpha}$, we get the above equality $(*)$ in the following form by writing down bimodules and intertwiners explicitly.

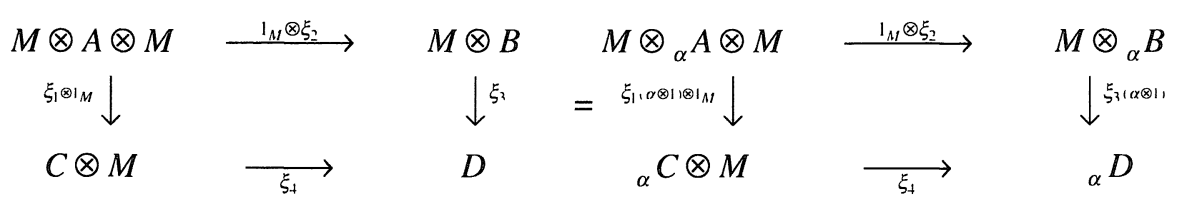

where $\xi$,'s satisfy one of the following

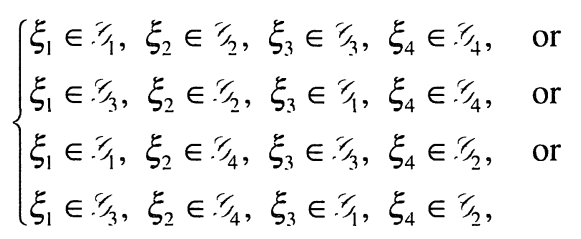


and $A=s\left(\xi_{2}\right)=s\left(\xi_{1}\right), B=r\left(\xi_{2}\right)=s\left(\xi_{3}\right), C=r\left(\xi_{1}\right)=s\left(\xi_{4}\right), D=r\left(\xi_{3}\right)=r\left(\xi_{4}\right)$.

The value of the left hand side of the equality is

$$
\xi_{4} \circ\left(\xi_{1} \otimes 1_{M}\right) \circ\left(1_{M} \otimes \xi_{2}\right)^{\prime} \circ\left(\xi_{3}\right),
$$

and that of the right hand side is

$$
\begin{aligned}
& \xi_{4} \circ\left(\xi_{1}(\alpha \otimes 1) \otimes 1_{M}\right) \circ\left(1_{M} \otimes \xi_{2}\right)^{\prime} \circ\left(\xi_{3}(\alpha \otimes 1)\right)^{\prime} \\
= & \xi_{4} \circ\left(\xi_{1} \otimes 1_{M}\right) \circ\left(\alpha \otimes 1 \otimes 1_{M}\right) \circ\left(\alpha \otimes 1 \otimes 1_{M}\right) \circ\left(1_{M} \otimes \xi_{2}\right) \circ\left(\xi_{3}\right) \\
= & \xi_{4} \circ\left(\xi_{1} \otimes 1_{M}\right) \circ\left(1_{M} \otimes \xi_{2}\right) \circ\left(\xi_{3}\right) .
\end{aligned}
$$

So the above equality holds.

(ii) In the case where some of the four bimodules $A, B, C, D$ are fixed by $\sigma_{\alpha}$, we will show the above equality when $B$ and $C$ are fixed points, and other cases are proved similarly. In this case, the equality $(*)$ is written in the following way.

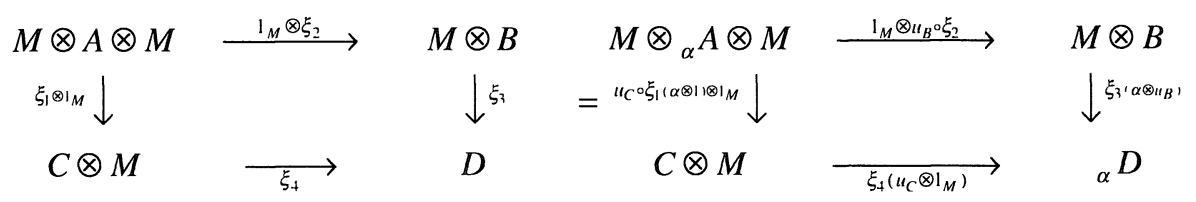

Here $u_{B}: B \rightarrow{ }_{\alpha} B$ and $u_{C}: C \rightarrow{ }_{\alpha} C$ are surjective isometry between these bimodules. The left hand side of the equality is

$$
\xi_{4} \circ\left(\xi_{1} \otimes 1_{M}\right) \circ\left(1_{M} \otimes \xi_{2}\right) \circ\left(\xi_{3}\right),
$$

and that of the right hand side is

$$
\begin{aligned}
& \xi_{4}\left(u_{C} \otimes 1_{M}\right) \circ\left(u_{C}^{\prime} \circ \xi_{1}(\alpha \otimes 1) \otimes 1_{M}\right) \circ\left(1_{M} \otimes u_{B}^{\prime} \circ \xi_{2}\right) \circ\left(\xi_{3}\left(\alpha \otimes u_{B}\right)\right)^{\prime} \\
= & \xi_{4}\left(u_{C} \otimes 1_{M}\right) \circ\left(u_{C} \circ \xi_{1}(\alpha \otimes 1) \otimes 1_{M}\right) \circ\left(1_{M} \otimes \xi_{2} \circ u_{B}\right) \circ\left(\left(\alpha \otimes u_{B} \xi_{3}\right)\right. \\
= & \xi_{4} \circ\left(\xi_{1} \otimes 1_{M}\right) \circ\left(\alpha \otimes 1 \otimes 1_{M}\right) \circ\left(\alpha \otimes 1 \otimes 1_{M}\right) \circ\left(1_{M} \otimes \xi_{2}\right)^{\prime} \circ\left(\xi_{3}\right)^{\prime} \\
= & \xi_{4} \circ\left(\xi_{1} \otimes 1_{M}\right) \circ\left(1_{M} \otimes \xi_{2}\right)^{\prime} \circ\left(\xi_{3}\right) .
\end{aligned}
$$

So the above equality holds.

Q.E.D.

Now we will give a necessary and sufficient condition for triviality of Loi's invariant of a non-strongly outer automorphism on a subfactor with a symmetric connection. 
Theorem 2.3. Let $N \subset M$ be a subfactor satisfying assumptions (A1) (A3) and (A6). As to the automorphism $\alpha \in \operatorname{Aut}(M, N)$, the following are equivalent for each $k \geq 0$.

(i) $\left.\quad \alpha\right|_{N^{\prime} \cap M_{\curlywedge}}=1_{N^{\prime} \cap M_{\curlywedge}}$.

(ii)

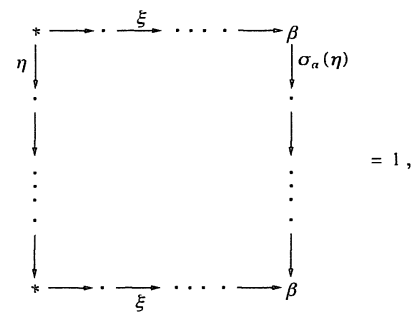

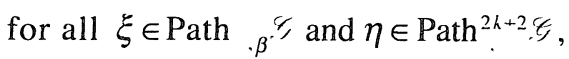

(iii) For all $\eta \in$ Path $^{2 h+2}$, , there exists a path $\xi \in$ Path $_{, \beta}$, such that

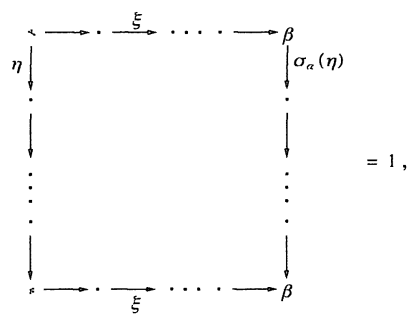

where the bimodules $*={ }_{N} N_{N}$ and $\beta={ }_{N}\left({ }_{\beta} N\right)_{N}$ and the symbol Path ${ }_{1,1}^{\prime}$ " represents the set of paths on the graph $\approx$ with source vertex $x$, range vertex $y$ and length $l$.

Proof. Before we prove this theorem, we claim that $\sigma_{\sigma}(\eta)=\eta(\alpha \otimes 1)$. We can easily show this by using the fact that the automorphism

$$
\underbrace{\alpha \otimes \alpha \otimes \cdots \otimes \alpha}_{n+1} \text { on } \underbrace{M \otimes_{N} M \otimes_{N} \cdots \otimes_{N} M}_{n+1}
$$

is the same as the one on $M_{n}$ extended by $\alpha\left(e_{l}\right)=e_{l}(i=1,2, \cdots, n)$ as above. (i) $\Rightarrow$ (ii). If $\left.\alpha\right|_{N^{\prime} \cap M_{h}}=1_{N^{\prime} \cap M_{h}}$, then we set

$$
\begin{aligned}
& \zeta \equiv \sigma_{\alpha}(\eta) \circ\left(1_{M_{h}} \otimes \xi\right)=\eta(\alpha \otimes 1) \circ\left(1_{M_{h}} \otimes \xi\right), \\
& \zeta^{\prime} \equiv \xi \circ\left(\eta \otimes 1_{M_{h}}\right),
\end{aligned}
$$

where $\xi \in$ Path $_{. \beta} "$ and $\eta \in \operatorname{Path}^{2 h+2 \%}$. If we denote $\eta(\hat{1})$ by $a \otimes \hat{1} \in_{N}\left(M_{h}\right)_{N} \otimes$ ${ }_{N}\left({ }_{\beta} N\right)_{N}$ and denote $\xi(\hat{1})$ by $\hat{1} \otimes b \in \epsilon_{N}\left({ }_{\beta} N\right)_{N} \otimes_{N}\left(M_{h}\right)_{N}$, where $\hat{1} \in N \cap \cap_{N}\left(L^{2}(N)\right)_{N}$, then 


$$
\begin{aligned}
\zeta(\hat{1}) & =\left(1_{M_{k}} \otimes \xi\right)\left(\alpha^{-1} \otimes 1\right) \eta(\hat{1})=\left(1_{M_{h}} \otimes \xi\right)\left(\alpha^{-1} \otimes 1\right)(a \otimes \hat{1}) \\
& =\left(1_{M_{k}} \otimes \xi^{\prime}\right)\left(\alpha^{-1}(a) \otimes 1\right)=\alpha^{-1}(a) \otimes \hat{1} \otimes b, \\
\zeta^{\prime \prime}(\hat{1}) & =\left(\eta^{\prime} \otimes 1_{M_{k}}\right) \xi^{\prime}(\hat{1})=\left(\eta \otimes 1_{M_{h}}\right)(\hat{1} \otimes b)=a \otimes \hat{1} \otimes b .
\end{aligned}
$$

And from the triviality of Loi's invariant $\alpha^{-1}(a)=a$ because $a \in N^{\prime} \cap M_{k}$ by Corollary 1.2. So $\zeta(\hat{1})=\zeta^{\prime \prime}(\hat{1})$ and hence $\zeta=\zeta^{\prime}$. So the value of the above partition function is one.

(ii) $\Rightarrow$ (iii) is trivial.

(iii) $\Rightarrow$ (i). We use the same notation $\zeta \equiv \eta(\alpha \otimes 1) \circ\left(1_{M_{k}} \otimes \xi\right)$ and $\zeta^{\prime} \equiv \xi \circ(\eta \otimes$ $1_{M_{\ell}}$ ). And if the equality (iii) holds, then we have

$$
\left\langle\zeta, \zeta^{\prime}\right\rangle=1,\|\zeta\|=1, \quad\left\|\zeta^{\prime}\right\|=1
$$

Hence $\zeta^{\prime}=\zeta$. So $\alpha^{-1}(a) \otimes_{N} b=a \otimes_{N} b$. This means $\alpha^{-1}(a)=a$, because $\alpha^{-1}(a)$ and $a$ are elements in higher relative commutants $N^{\prime} \cap M_{h}$ and if $x e_{N}^{M_{k}} b=0$ and $b \neq 0, x \in N^{\prime} \cap M_{h}$ then $x=0$. Indeed, if $x e_{N}^{M_{h}} b=0$ then

$$
\begin{aligned}
b^{\prime} e_{N}^{M_{\curlywedge}} x x e_{N}^{M_{\curlywedge}} b & =b E_{N}^{M_{\curlywedge}}\left(\begin{array}{ll}
x & x
\end{array}\right) e_{N}^{M_{\curlywedge}} b \\
& =b \operatorname{tr}(x x) e_{N}^{M_{\curlywedge}} b=0 .
\end{aligned}
$$

So $x=0$. Hence $\alpha^{-1}(a)=a$ for all $a \in N^{\prime} \cap M_{k}$ such that $a=\eta(\hat{1}), \eta \in \operatorname{Path}^{2 h+2}$, . Since for all $x \in N^{\prime} \cap M_{k}$ there exists an intertwiner $\xi \in \operatorname{Hom}\left({ }_{N}\left(M_{h}\right)_{N},{ }_{N} N_{N}\right)$ by Corollary 1.2 and these intertwiners in $\operatorname{Hom}\left({ }_{N}\left(M_{h}\right)_{N}{ }_{N} N_{N}\right)$ are linear combinations of the basis $\operatorname{Path}^{2 h+2}$, , we have $\alpha^{-1}(a)=a$ for all $a \in N^{\prime} \cap M_{h}$. Q.E.D.

Remark. The same partition function was used in [EK2] page 363 to get the triviality of the Loi's invariant in the AFD case, but their method works only in the AFD case.

\section{§3. Orbifold Subfactors of the Non-AFD $S U(n)_{h}$ Subfactors}

In this section we will apply the result of the previous section to non-AFD $S U(n)_{h}$ subfactors. In this paper if a non-AFD subfactor $N \subset M$ has the same paragroup (higher relative commutants) as an AFD $S U(n)_{h}$ subfactor $P \subset Q$, then we call it a non-AFD $S U(n)_{k}$ subfactor. (We mean by $S U(n)_{k}$ subfactors the subfactors arising from $S U(n)_{h}$ WZW models as in [BG].) We consider the following non-AFD $S U(n)$ subfactors with level $k$.

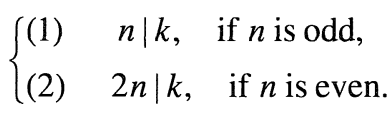


In this case these subfactors satisfy conditions (A1) (A3) and (A6) and have $\mathbb{Z}_{n}$ symmetric connections as in $[E K 1],[X 1]$. So we can construct the orbifold subfactors $N \rtimes_{\alpha} \mathbb{Z}_{n} \subset M \rtimes_{\alpha} \mathbb{Z}_{n}$. If we make a symmetric choice of bimodules and intertwiners, the following partition functions become well-defined, because the values do not depend on the gauge choice any more. So we can show the following proposition in the same way as in [X1].

Proposition 3.1. (Xu [X1]) For each $\mathbb{Z}_{n}$ symmetric connection $W$ on a non-AFD $S U(\mathrm{n})$ subfactor with level $k$ such that $n \mid k$, if $n$ is odd, or $2 n \mid k$, if $n$ is even, we have the following equalities.

(i)

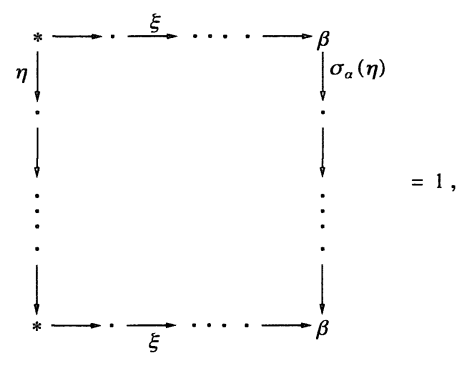

for all $\xi \in$ Path $\beta$, and $\eta \in \operatorname{Path}^{2 /+2}$, where the bimodules $*={ }_{N} N_{N}$ and $\beta={ }_{N}\left({ }_{\beta} N\right)_{N}$.

(ii)

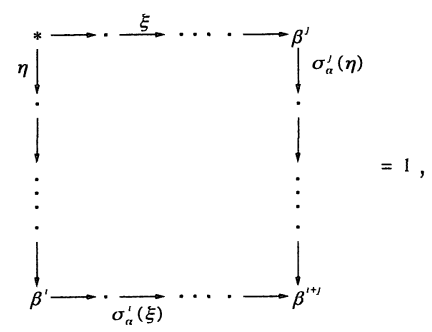

for all $\xi \in$ Path ${ }_{\beta^{\prime}} \%$ and $\eta \in \operatorname{Path}_{\beta^{\prime}}^{2 l+2} \%$.

(iii)

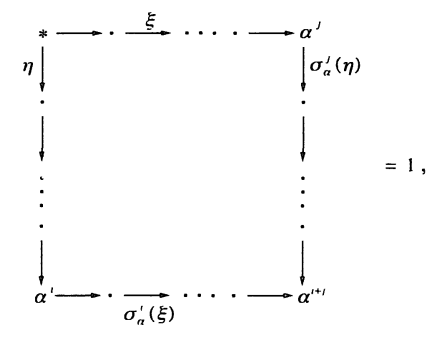

for all $\xi \in$ Path ${ }_{. \alpha^{\prime}} \not{H}$ and $\eta \in \operatorname{Path}^{2 \prime}{ }_{* \alpha^{\prime}} \mathscr{H}$. Here the bimodules $*={ }_{M} M_{M}$ and $\alpha={ }_{M}\left({ }_{\alpha} M\right)_{M}$. 
Now we can compute the higher relative commutants of the orbifold subfactor $N \rtimes_{\alpha} \mathbf{Z}_{n} \subset M \rtimes_{\alpha} \mathbf{Z}_{n}$, i.e., $\left(N \rtimes_{\alpha} \mathbf{Z}_{n}\right)^{\prime} \cap\left(M_{l} \rtimes_{\alpha} \mathbf{Z}_{n}\right)$. If we take $\sum_{l=0}^{n-1} x_{l} u^{\prime} \in$ $\left(N \rtimes_{\alpha} \mathbb{Z}_{n}\right)^{\prime} \cap\left(M_{l} \rtimes_{\alpha} \mathbb{Z}_{n}\right), x_{l} \in M_{l}, \operatorname{Ad} u=\alpha$, then $x_{l}$ 's satisfy the following,

$$
\left\{\begin{array}{l}
x_{0} \in\left(N^{\prime} \cap M_{l}\right)^{\alpha}=N^{\prime} \cap M_{l}, \\
x_{t} \in M_{l}, \quad n x_{t}=x_{l} \alpha^{\prime}(n) \text { for all } n \in N, \text { and } \alpha\left(x_{l}\right)=x_{l},(1 \leq i \leq n-1),
\end{array}\right.
$$

where the first equality $\left(N^{\prime} \cap M_{l}\right)^{\alpha}=N^{\prime} \cap M_{l}$ holds because Loi's invariant of the automorphism $\alpha$ is trivial from Proposition 3.1 (i) and Theorem 2.3.

If the strong outerness of the automorphism $\alpha \in \operatorname{Aut}(M, N)$ breaks at the $r$-th extension $M_{1}$, then the following hold from Theorem 1.1 .

$$
\begin{cases}x_{l}=0 & (l=0,1,2, \cdots, r-1), \\ \text { there exists a non-zero element } x_{t} \in M, & (l=r),\end{cases}
$$

When $l=r$, the above $x_{1}$ can be represented by the linear combination of a's such that $a=\eta^{\prime}(\hat{1}), \eta \in \mathrm{Path}_{\beta^{\prime}}^{2 h+2}$. From the computation of the partition function of (ii) in Proposition 3.1, $\alpha^{-1}\left(x_{t}\right)=x_{t}$ for all $j \in \mathbf{N}$. Hence $\alpha\left(x_{t}\right)=x_{t}$. So the higher relative commutants increase at the $r$-th level $\left(N \rtimes_{\alpha} \mathbf{Z}_{n}\right)^{\prime} \cap M, \rtimes_{\alpha} \mathbf{Z}_{n}$ in the same way as in the AFD case. Similarly we can compute the higher relative commutants $\left(M \rtimes_{\alpha} \mathbf{Z}_{n}\right)^{\prime} \cap M_{l} \rtimes_{\alpha} \mathbf{Z}_{n}$ by using the equality (iii) in the previous proposition.

Now we have completed a proof of the following theorem.

Theorem 3.2. Let $N \subset M$ be a non-AFD $S U(n)$ subfactor with level $k$ which has the same paragroup as the $A F D S U(n)_{k}$ subfactor $P \subset Q$. Here $k$ satisfies the following; $n \mid k$, if $n$ is odd, or $2 n \mid k$, if $n$ is even. And let $\alpha$ be the automorphism in $\operatorname{Aut}(M, N)$ satisfying conditions (A2) and (A3). Then $\alpha$ induces a $\mathbf{Z}_{n}$-action on this subfactor $N \subset M$, and we can construct the orbifold subfactor $N \rtimes_{\alpha} \mathbf{Z}_{n} \subset M \rtimes_{\alpha} \mathbf{Z}_{n}$. The paragroup of this new subfactor coincides with that of the AFD orbifold subfactor $P \rtimes_{\alpha} \mathbb{Z}_{n} \subset Q \rtimes_{\alpha} \mathbf{Z}_{n}$.

F. Xu extended [X1] to the case of AFD $S U(n)$ subfactors with level $k$ such that $n \mid k$ and $2 n \nmid k$ in [X2]. And we get a similar extension of the Theorem 3.2 as follows.

Proposition 3.3. (Xu [X2]) For each $\mathbb{Z}_{n}$ symmetric connection $W$ on non-AFD $S U(n)$ subfactor with level $k$ such that $n \mid k$ and $n=2 n^{\prime}$ is even, we have the following equalities. We set $k=n k^{\prime}$. 
(i)

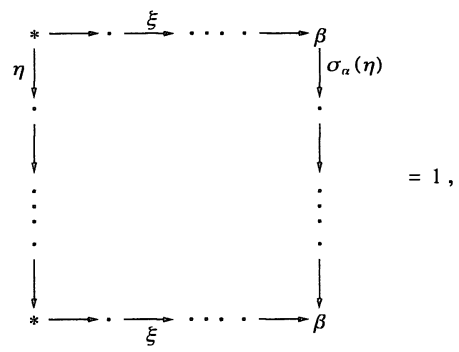

for all $\xi \in$ Path ${ }_{, \beta} \mathscr{G}$ and $\eta \in \operatorname{Path}^{21+2} \mathscr{G}$, where the bimodules $*={ }_{N} N_{N}$ and $\beta=$ ${ }_{N}\left({ }_{\beta} N\right)_{N}$.

(ii)

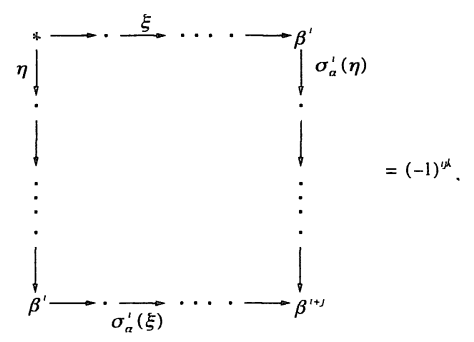

for all $\xi \in$ Path $_{, \beta^{\prime}}$, and $\eta \in \operatorname{Path}_{\beta^{\prime}}^{21+2 \xi}$.

(iii)

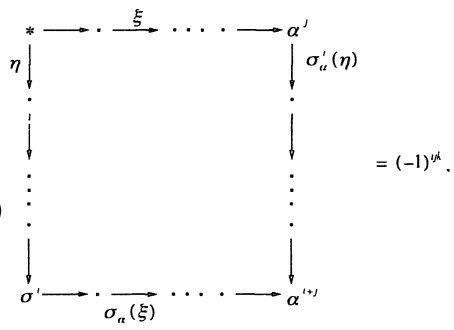

for all $\xi \in$ Path ${ }_{. \alpha^{\prime}} G$ and $\eta \in$ Path $_{. \alpha^{\prime}}^{2 l} \geqslant$. Here the bimodules $*={ }_{M} M_{M}$ and $\alpha=$ ${ }_{M}\left({ }_{\alpha} M\right)_{M}$.

From this Proposition, in the case of non-AFD $S U(n)$ subfactor $N \subset M$ with level $k$ such that $n \mid k, n$ is even and $2 n \nmid k$, we also construct an orbifold subfactor. In this case $N \rtimes_{\alpha} \mathbb{Z}_{2 n^{\prime}} \subset M \rtimes_{\alpha} \mathbb{Z}_{2 n^{\prime}}$ and $N \rtimes_{\alpha^{\prime}} \mathbb{Z}_{n^{\prime}} \subset M \rtimes_{\alpha^{\prime}} \mathbb{Z}_{n^{\prime}}$ have the same paragroups because of the following argument.

If we take $\sum_{l=0}^{n-1} x_{l} u^{\prime} \in\left(N \rtimes_{\alpha} \mathbb{Z}_{n}\right)^{\prime} \cap\left(M_{l} \rtimes_{\alpha} \mathbb{Z}_{n}\right), x_{1} \in M_{l}, \operatorname{Ad} u=\alpha$, then $x_{1}$ 's satisfy the following,

$$
\left\{\begin{array}{l}
x_{0} \in\left(N^{\prime} \cap M_{l}\right)^{\alpha}=N^{\prime} \cap M_{l}, \\
x_{l} \in M_{l}, n x_{l}=x_{l} \alpha^{\prime}(n) \text { for all } n \in N, \text { and } \alpha\left(x_{l}\right)=x_{l},(1 \leq i \leq n-1),
\end{array}\right.
$$

where the first equality $\left(N^{\prime} \cap M_{l}\right)^{\alpha}=N^{\prime} \cap M_{l}$ also holds because Loi's invariant of the automorphism $\alpha$ is trivial from Proposition 3.3 (i) and Theorem 2.3. 
If the strong outerness of the automorphism $\alpha \in \operatorname{Aut}(M, N)$ breaks at the $r$-th extension $M_{1}$, then the following hold from Theorem 1.1 .

$$
\begin{cases}x_{i}=0 & (l=0,1,2, \cdots, r-1), \\ \text { there exists a non - zero element } x_{t} \in M, & (l=r) .\end{cases}
$$

When $l=r$, from the computation of the partition function of (ii) in Proposition 3.3, $\alpha^{-1}\left(x_{l}\right)=(-1)^{\prime \prime} x_{\imath}$ for all $j \in \mathbb{N}$. Hence $\alpha\left(x_{l}\right)=(-1)^{\prime} x_{l}$. This means $\alpha\left(x_{2 l}\right)$ $=x_{2 l}$. And if we take $\sum_{\jmath=0}^{n-1} x_{2,} u^{\prime \prime} \in\left(N \rtimes_{\alpha^{2}} \mathbb{Z}_{n^{\prime}}\right)^{\prime} \cap\left(M_{l} \rtimes_{\alpha^{2}} \mathbb{Z}_{n^{\prime}}\right), x_{2}, \in M_{l}, \quad \operatorname{Ad} u=\alpha$, similarly, we have $\alpha^{2}\left(x_{2}\right)=x_{2}$, when $l=r$. So these two subfactors have the same higher relative commutants. For the dual principal graph, we can get the similar conclusion by using the equality (iii) in Proposition 3.3.

Thus we have the following theorem.

Theorem 3.4. Let $N \subset M$ be a non-AFD $S U(n)$ subfactor with level $k$ which has the same paragroup as a $A F D S U(n)_{k}$ subfactor $P \subset Q$. Here $n$ satisfies the following; $n \mid k, 2 n \nmid k$ and $n=2 n^{\prime}$ is even. And let $\alpha$ be an automorphism in $\operatorname{Aut}(M, N)$ satisfying the condition (A2) and (A3). Then $\alpha$ induces a $\mathbb{Z}_{n}$-action on this subfactor $N \subset M$, and we can construct the orbifold subfactor $N \rtimes_{\alpha} \mathbb{Z}_{n} \subset M \rtimes_{\alpha} \mathbb{Z}_{n}$ and $N \rtimes_{\alpha^{2}} \mathbb{Z}_{n^{\prime}} \subset M \rtimes_{\alpha^{2}} \mathbb{Z}_{n^{\prime}}$.These two subfactors have the same paragroups. And the paragroups of these new subfactors coincide with that of the AFD orbifold subfactors $P \rtimes_{\sigma} \mathbb{Z}_{n} \subset Q \rtimes_{\alpha} \mathbb{Z}_{n}$ and $P \rtimes_{\alpha^{2}} \mathbb{Z}_{n^{\prime}} \subset Q \rtimes_{\alpha^{2}} \mathbb{Z}_{n^{\prime}}$.

This is a generalization of Xu's theorem in [X2] for the AFD case, and our proof is simpler than that of [X2] even in the AFD case.

If we think of the case where $n=2$ in the above theorem, we conclude that the orbifold subfactors of non-AFD subfactors of type $I_{1}$ with principal graphs $A_{4 m-1}$ have the same principal graphs. This is a part of the result in Theorem 3.9 of $[\mathrm{G}]$.

\section{Comment.}

S. Popa and F. Radulescu showed existence of non-AFD $S U(n)_{h}$ subfactors of type $\mathrm{II}_{1}$ whose paragroups are the same as that of AFD $S U(n)_{k}$ subfactors of type $\mathrm{II}_{1}$ in [P3], [R]. So we can apply our result to these subfactors. Their subfactors are not isomorphic to $N \otimes P \subset M \otimes P$ where $N \subset M$ is of AFD $S U(n)_{k}$ subfactors of type $\mathrm{II}_{1}$ and $P$ is an arbitrary non-AFD factor of type $\mathrm{II}_{1}$.

\section{References}

[BG] de Boer, J. and Goeree, J., Markov traces and $\mathrm{II}_{1}$ factors in conformal field theory, $C \mathrm{com}$. Math. Phy's., 139 (1991), 267-304. 
[CK] Choda, M. and Kosaki, H., Strongly outer actions for an inclusion of factors, J. Funct. Anal., 122 (1994), 315-332.

[C] Connes, A., Periodic automorphisms of the hyperfinite factor of type $\mathrm{II}_{1}$, Acta Sci. Math., 39 (1977), 39-66.

[EK1] Evans, D.E. and Kawahigashi, Y., Orbifold subfactors from Hecke algebras, Comm. Math. Phys., 165 (1994), 445-484.

[EK2] Subfactors and conformal field theory, Quantum and non-commutative analysis, 341-369, Kluwer Academic (1993).

[G] Goto, S., Orbifold construction for non-AFD subfactors, Int. J. of Math., 5 (1994), 725-746.

[I] Izumi, M., Goldman's type theorem for index 3, Publ.RIMS, Kyoto Univ., 28 (1992), $833-$ 843 .

[Kal] Kawahigashi, Y., On flatness of Ocneanu's connections on the Dynkin diagrams and classification of subfactors, J. Funct. Anal., 127 (1995), 63 -107.

[Ka2] Centrally trivial automorphisms and an analogue of Connes's $\chi(M)$ for subfactors, Duke Math. J., 71 (1993), 93-118.

[Ko] Kosaki, H., Automorphisms in the irreducible decompositions of sectors, Quantum and noncommutative analysis, 305-316, Kluwer Academic (1993).

[L] Loi, P. H., On automorphisms of subfactors, preprint, 1990.

[O1] Ocneanu, A., Quantized group string algebras and Galois theory for algebras, Operator algebras and applications, 2 (Warwick, 1987), London Math. Soc. Lect. Note Series 136 , Cambridge University Press, (1988), 119-172.

[O2] Q Quantum symmetry, differential geometry of finite graphs and classification of subfactors, University of Tokyo Seminary Notes 45, (Notes recorded by Y. Kawahigashi), 1991.

[O3] An invariant coupling between 3-manifolds and subfactors, with connections to topological and conformal quantum field theory, preprint, 1991.

[P1] Popa, S., On the classification of actions of amenable groups on subfactors, C. R. Acad. Sc. Paris., 315 (1992), 295-299.

[P2] Classification of actions of discrete amenable groups on amenable subfactors of type II, preprint, 1992.

[P3] An axiomatization of the lattice of higher relative commutants of a subfactor, Invent. Math., 120 (1995), 427-446.

[R] Rādulescu, F., Random matrices, amalgamated free products and subfactors of the von Neumann algebra of a free group of noninteger index, Invent. Math., 115 (1994), 347-389.

[X1] Xu, F., Orbifold construction in subfactors, Comm. Math. Phys., 166 (1994), 237-253.

[X2] The flat part of non-flat orbifolds (1993), to appear in Pac.J.of Math.

[Y1] Yamagami, S., A note on Ocneanu's approach to Jones' index theory, Internat. J. of Math., 4 (1993), 859-871.

[Y2] , Modular theory for bimodules, J. Funct. Anal., 125 (1994), 327-357. 